\title{
A Practical Approach For Excess Bandwidth Distribution for EPONs
}

\author{
Amr Elrasad and Basem Shihada \\ CEMSE, King Abdullah University of Science And Technology (KAUST), Saudi Arabia. \\ amr.rasad@kaust.edu.sa,basem.shihada@kaust.edu.sa
}

\begin{abstract}
This paper introduces a novel approach, DES, to divide excess bandwidth in EPONs. DES is more suitable to industrial deployment as it requires no timing constraints and it achieves better performance than the previously reported schemes.
\end{abstract}

OCIS codes: $060.1155,060.4256$.

\section{Introduction}

Fiber-to-the-premises (FTTP) has been proposed as a solution to increasing bandwidth demand in access networks. FTTP aims to deeply penetrate optical fiber to the user premises. Passive Optical Network (PON) is considered one of the main promising approaches to apply FTTP in the first mile. PON is popular for many reasons: it uses passive components between central office (CO) and user premisses; it can support remote users over long distances up to $20 \mathrm{Km}$ in legacy PONs and $100 \mathrm{Km}$ in long-reach PONs (LR-PON). There exists two approaches for PON: Ethernet Passive Optical Network (EPON), standardized by IEEE; Gigabit-Capable Passive Optical Networks (GPON), standardized by ITU.

Bandwidth allocation (BA) is considered the most vital task in EPONs. It determines when optical network units (ONUs) are permitted to send their upstream data to the optical line terminal (OLT). Bandwidth allocation can be static (SBA) or dynamic (DBA). DBA is more suitable for access networks due to its bursty traffic nature. The 802.3ah task force decided that BA is left to be a vendor specific job. There have been many research contributions to the DBA problem in the last decade ranging from simplified algorithms to too complex ones. The efficiency of DBA depends on bandwidth utilization which in turn reduces the average packet delay.

Interleaved polling with adaptive cycle time [1] is One of the benchmark DBA schemes. The OLT assigns bandwidth on the fly to ONUs. The bandwidth assignment can be gated,limited, elastic, linear credit, or constant credit. The limited approach is the most popular one as it limits the cycle time to a maximum threshold which makes it a suitable scheme for differentiated services (DiffServ) EPON. In IPACT-Limted, ONU $i$ is granted no more than $B_{i}^{\min }$ bytes during each cycle representing the minimum guaranteed bandwidth. The granted bandwidth in cycle $n, B_{i}(n)$, is given by $B_{i}(n)=\min \left(R_{i}(n), B_{i}^{\min }\right)$, where $R_{i}(n)$ is the reported ONU buffer size.

The concept of excess bandwidth distribution [2] was introduced to increase bandwidth utilization. The ONUs were divided into underloaded and overloaded according to the reported buffer size. The authors proposed two schemes DBA1 and DBA2. DBA1 waits to receive report messages from all ONUs before redistributing the excess bandwidth among overloaded ONUs. This mechanism incorporates idle time, $T_{i d l e}=R T T+T_{D B A}$, between cycles and hence degrades the bandwidth utilization. BDA2 assigns underloaded ONUs their bandwidth grant on the fly and postpones overloaded ONUs till reception of all report messages. DBA2 still suffers from $T_{i d l e}$ at high load. Both DBA1 and DBA2 use uncontrolled excess allocation, this means that some overloaded ONUs may be granted bandwidth more than requested. This problem was solved in [3]. The authors presented iterative scheme (W-DBA) to effectively distributed the excess bandwidth. In order to solve the problem of $T_{i d l e}$, Zheng [4] proposed an early allocation scheme (E-DBA2) to remove $T_{\text {idle }}$. The proposed scheme keeps track of last assigned grant end time, $t_{\text {end }}$, and the current time, $t$. If $t_{\text {end }}-t<T_{\text {idle }}$, The OLT will schedule one of the overloaded ONUs without assigning excess bandwidth to it. one of the proposed scheme drawbacks is its need of precise timing to remove $T_{i d l e}$ completely. The scheme might introduce some idle time if the gate message was delayed by a long ethernet downstream frame.

With the emergence of EPON technology, the deployment of 10G-EPON and the increased number of ONUs per EPON, the problem of idle time removal is becoming more persistent. This paper introduces a simplified yet very effective solution to both uncontrolled allocation and idle time problems. The proposed scheme, Delayed Excess Scheduling (DES), is more suitable for industrial deployment due to its simplicity and its superior performance against DBA2 and E-DBA2. 


\section{Delayed Excess Scheduling}

DES main idea is to delay scheduling of excess bandwidth to the next cycle and use the assigned excess bandwidth as increase to $B_{i}^{\min }$. In other words, the maximum assigned bandwidth during cycle $n$ is $B_{i}^{\min }(n)=B_{i}^{\min }+E_{i}(n-1)$, where $E_{i}(n-1)$ is the excess bandwidth share of ONU $i$ from cycle $(n-1)$. DES divides ONUs into 3 groups:

1. Underload (U): an ONU is considered underloaded if $R_{i}(n) \leq B_{i}^{\min }$

2. Satisfied (S): an ONU is satisfied if $B_{i}^{\min }<R_{i}(n) \leq B_{i}^{\min }(n)$

3. Overloaded (O): an ONU is considered overloaded if $R_{i}(n)>B_{i}^{\min }(n)$

The algorithm works as follows:

- During cycle $(n-1)$, The OLT receives bandwidth requests for cycle $n$.

- The OLT assigns on the fly bandwidth grant $B_{i}(n)=\min \left(R_{i}(n), B_{i}^{\min }(n)\right)$.

- The OLT identify the state of each ONU: U, S, or O.

- After reception of all report messages, The OLT calculates the excess bandwidth share of each overloaded ONU as $E_{i}(n)=\frac{w_{i}}{\sum_{k \in O} w_{j}} \sum_{j \in U} B_{i}^{\text {min }}-R_{i}(n)$, where $w_{i}$ is the weight assigned to each ONU according to service level agreement.

- The OLT updates $B_{i}^{\min }(n+1)$ as

$$
B_{i}^{\text {min }}(n+1)= \begin{cases}B_{i}^{\text {min }}+E_{i}(n) & i \in O \\ B_{i}^{\text {min }} & i \in S \cup U .\end{cases}
$$

It can be seen that DES does not incorporate any idle time and does not allocate overloaded ONUs unnecessary bandwidth. DES is very simple and does not require any precise timing procedure and can be implemented easily. The next section will show how effective DES are against the benchmark DBA schemes.

\section{Simulation and Numerical Results}

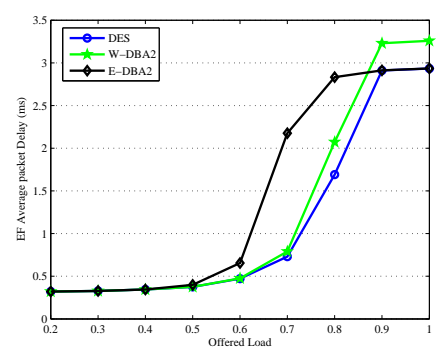

(a)

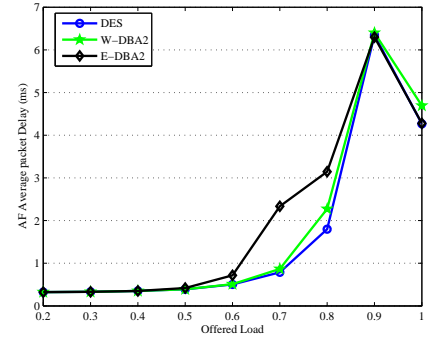

(b)

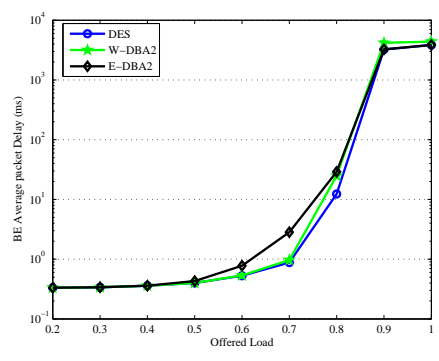

(c)

Fig. 1: Average packet delay: (a) EF (b) AF (c) BE

We Consider an EPON with a single OLT and 16 ONUs with 10MB buffer size. The upstream and downstream transmission rates are symmetric with $1 \mathrm{~Gb} / \mathrm{s}$. We use the packet size distribution reported in [5], with a minimum IFG of 12 bytes and preamble of 8 bytes. The distance between OLT and all ONUs is set to 20Km, which corresponds to $200 \mu \mathrm{sec}$ RTT. The generated traffic is DiffServ with 3 classes of service: Expedited Forward (EF), Assured Forward (AF), and Best Effort (BE). EF ONU offered load share is 20\%, while the rest is divided equally between AF and BE. EF is constant-bit-rate (CBR) traffic with Possion arrivals and fixed packet size of 70 bytes, while AF and BE traffic are self-similar with long range dependence and hurst parameters $0.8 . B_{i}^{\min }$ is set to 15000 bytes and the guard 
bandwidth is set to 625 bytes. DES is evaluated against E-DBA2 [4] and a combination of DBA-2 [2] and W-DBA [3].

Figure1 shows the average packet delay for EF, AF, and BE traffic. It can be seen that DES shows superior performance against E-DBA2 and W-DBA2. DES reduces the EF average delay by 1.5 ms compared to E-DBA2 at 0.7 load and by $0.4 \mathrm{~ms}$ compared to W-DBA2 at 0.8 load. At load beyond 0.9, we notice that DES and E-DBA2 are similar while W-DBA2 is lagging due to idle time problem. The reason behind DES excel is its ability to remove idle time and incorporating controlled excess allocation as well. DES shows similar performance in both AF and BE traffic. For AF, DES reduces the average delay by $1.1 \mathrm{~ms}$ and $0.3 \mathrm{~ms}$ compared to E-DBA2 and W-DBA2 respectively at 0.8 load. At 0.8 load, DES reduces the average delay by $20 \mathrm{~ms}$ for BE traffic.

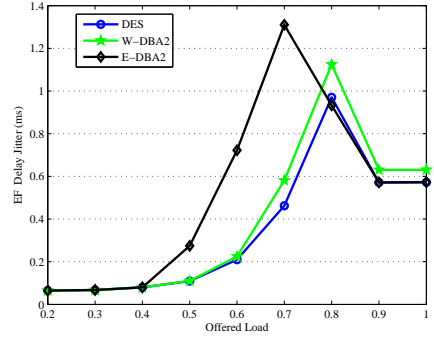

(a)

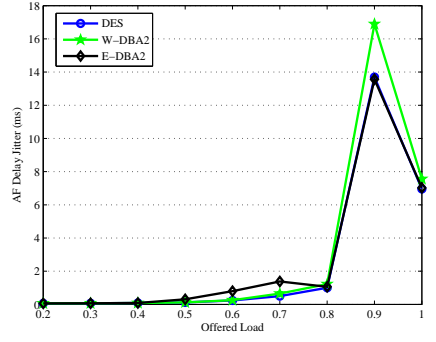

(b)

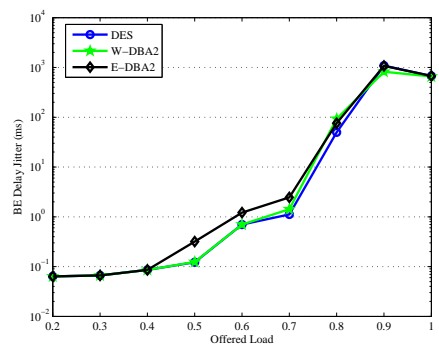

(c)

Fig. 2: Average delay jitter: (a) EF (b) AF (c) BE

Another important performance measure for delay senstive traffic is delay jitter. Delay jitter is shown in figure2. For EF traffic, DES jitter reduction ranges from $0.1 \mathrm{~ms}$ to $0.8 \mathrm{~ms}$ at load 0.5-0.7 compared to E-DBA2. The EF jitter of DES and W-DBA2 are close with advantage to DES. For AF traffic, both DES and W-DBA2 Jitter are lower than E-DBA2 at 0.5-0.8 load. At load 0.8-full load, DES and E-DBA2 are better than W-DBA2. The performance of the 3 schemes is close in case of BE with relative advantage to DES.

\section{References}

1. G. Kramer, B. Mukherjee, and G. Pesavento, "Interleaved polling with adaptive cycle time (IPACT): a dynamic bandwidth distribution scheme in an optical access network," Photonic Network Communications 4, 89-107 (2002).

2. C. M. Assi, Y. Ye, S. Dixit, and M. A. Ali, "Dynamic bandwidth allocation for quality-of-service over Ethernet PONs," Selected Areas in Communications, IEEE Journal on 21, 1467-1477 (2003).

3. X. Bai, A. Shami, and C. Assi, "On the fairness of dynamic bandwidth allocation schemes in Ethernet passive optical networks," Computer Communications 29, 2123-2135 (2006).

4. J. Zheng, "Efficient bandwidth allocation algorithm for Ethernet passive optical networks," IEE ProceedingsCommunications 153, 464-468 (2006).

5. D. Sala and A. Gummalla, "PON functional requirements: services and performance," IEEE 802, 9-13 (2001). 